\title{
Modulation of neural oscillations by vagus nerve stimulation in posttraumatic multifocal epilepsy: case report
}

\author{
Adeel Ilyas, MD, ${ }^{1,3}$ Emilia Toth, PhD, ${ }^{2,3}$ Diana Pizarro, BSEE, ${ }^{2,3}$ Kristen O. Riley, MD, ${ }^{1}$ and \\ Sandipan Pati, MD ${ }^{2,3}$
}

Departments of ${ }^{1}$ Neurosurgery and ${ }^{2}$ Neurology; and ${ }^{3}$ Epilepsy and Cognitive Neurophysiology Laboratory, University of Alabama at Birmingham, Alabama

\begin{abstract}
The putative mechanism of vagus nerve stimulation (VNS) for medically refractory epilepsy is desynchronization of hippocampal and thalamocortical circuitry; however, the nature of the dose-response relationship and temporal dynamics is poorly understood. For greater elucidation, a study in a nonepileptic rat model was previously conducted and showed that rapid-cycle (RC) VNS achieved superior desynchrony compared to standard-cycle (SC) VNS. Here, the authors report on the first in-human analysis of the neuromodulatory dose-response effects of VNS in a patient with posttraumatic, independent, bilateral mesial temporal lobe epilepsy refractory to medications and SC-VNS who was referred as a potential candidate for a responsive neurostimulation device. During stereotactic electroencephalography (SEEG) recordings, the VNS device was initially turned off, then changed to SC-VNS and then RC-VNS settings. Spectral analysis revealed a global reduction of power in the theta $(4-8 \mathrm{~Hz})$ and alpha $(8-15 \mathrm{~Hz})$ bands with both SC- and RC-VNS compared to the stimulation off setting $(p<0.001)$. Furthermore, in the alpha band, both SC- and RC-VNS were associated with greater global desynchrony compared to the off setting $(p<0.001)$; and, specifically, in the bilateral epileptogenic hippocampi, RC-VNS further reduced spectral power compared to SC-VNS $(p<0.001)$. The dose-response and temporal effects suggest that VNS modulates regional and global dynamics differently.
\end{abstract}

https://thejns.org/doi/abs/10.3171/2018.6.JNS18735

KEYWORDS vagus nerve stimulator; epilepsy; mechanism; rapid cycle; case report

$\mathrm{V}$ AGUS nerve stimulation (VNS) can modulate dysfunctional circuits associated with select neurological and psychiatric disorders., ${ }^{5,919}$ Previous studies have demonstrated variable patient outcomes and side effects with different stimulation duty cycles; however, the neural dynamic underpinnings of this variability remain poorly elucidated. ${ }^{7,10,16}$ To increase understanding, a preclinical study in a nonepileptogenic rat model was conducted and showed that rapid-cycle VNS modulated hippocampal field spectral power and synchrony. ${ }^{17}$ Capitalizing on an intracranial electroencephalography (EEG) investigation performed as a workup for epilepsy surgery, we report the first in-human study demonstrating VNS duty-cycle modulation of network dynamics at meso and macro scales.

\section{Case Report}

Patient History

A 37-year-old, right-handed male was referred to our level 4 epilepsy center for management of focal epilepsy that had been refractory to five antiseizure medications. At the age of 18 , four years after a major trauma resulting in right temporal encephalomalacia, the patient developed focal seizures (2-4 seizures/month) that occasionally progressed to bilateral tonic-clonic seizures (1 seizure every 4-6 months). Seizure semiology consisted of impaired awareness, bilateral manual automatisms followed by confusion, and paraphasic errors. A VNS device (AspireSR model 106, LivaNova) was implanted at an outside facility and was initially effective for a year with a reduction in seizure frequency and severity; however, increased seizures and an episode of status epilepticus occurred later, prompting the referral.

Based on the clinical presentation and phase I investigations including scalp video-EEG, magnetoencephalography, structural evaluations (3-T MRI), and metabolic investigations (FDG-PET), a consensus was reached in our multidisciplinary epilepsy conference to perform robot-assisted (ROSA device, Medtech) stereotactic EEG

ABBREVIATIONS EEG = electroencephalography; $\mathrm{fMRI}$ = functional MRI; NREM = non-rapid eye movement; $\mathrm{POD}=$ postoperative day; $\mathrm{RC}=$ rapid cycle; $\mathrm{SC}=$ standard cycle; $S E E G$ = stereotactic EEG; VNS = vagus nerve stimulation.

SUBMITTED March 16, 2018. ACCEPTED June 12, 2018.

INCLUDE WHEN CITING Published online November 16, 2018; DOI: 10.3171/2018.6.JNS18735. 
(SEEG) electrode implantation into the bilateral hippocampi, amygdala, orbitofrontal gyri, anterior cingulate gyri, and right anterior insula to identify seizure foci (Fig. 1A). Overall, five electroclinical seizures were identified, three of which originated from the right and two from the left amygdala-hippocampi. These seizures rapidly propagated to the bilateral orbitofrontal gyri. Wada testing demonstrated memory support from bilateral hippocampi; therefore, the decision was made to treat the bilateral hippocampal seizures with the FDA-approved responsive neurostimulation and to consider unilateral anterior temporal lobectomy in the future based on dominant seizure laterality derived from chronic ambulatory electrocorticography.

\section{SEEG Electrode Implantation and Data Acquisition}

A total of 166 electrodes were implanted and recorded at $2048 \mathrm{~Hz}$ using the Natus Xltek EEG system (Quantum). Scalp EEG activity from the midline $(\mathrm{Cz}, \mathrm{Pz})$ and frontocentral (F3, F4, C3, C4) regions was recorded simultaneously. Following SEEG electrode implantation, VNS was turned off on postoperative day (POD) 1 (Fig. 1B). The VNS was activated to standard cycle (SC; $2.5 \mathrm{~mA}, 25-\mathrm{Hz}$ signal frequency, $250-\mu$ sec pulse width, 60 -second stimulation on time, 108-second stimulation off time, duty cycle $38 \%$ ) on POD 5 and then switched to rapid cycle (RC; 2.5 $\mathrm{mA}, 25-\mathrm{Hz}$ signal frequency, $250-\mu \mathrm{sec}$ pulse width, 7-second on time, 18-second off time, duty cycle 44\%) on POD 6 , which was continued after discharge from the hospital. The decision to switch to RC was based on the partial response to seizure control with SC, and informed patient consent was obtained prior to this switch.

For each of the three VNS settings (i.e., off, SC, and $\mathrm{RC}$ ), representative samples in the latter half of a seizurefree 24-hour recording were obtained during the awake and non-rapid eye movement (NREM) sleep states, thus yielding a total of 6 EEG samples. From these 6 representative samples, 20 randomly selected 20 -second samples were selected for analysis. The criteria for selection of the representative samples were as follows. All samples were collected at least 8 hours following a VNS setting change. Simultaneous scalp video-EEG was used to identify appropriate awake and sleep states. Awake samples were obtained when the patient was resting motionlessly with a prominent underlying beta or alpha rhythm. Sleep samples were obtained during slow-wave NREM sleep. ${ }^{22}$ Based on these criteria, a total of 20,15, and 15 minutes of EEG data were obtained for the awake stimulation off, SC, and RC states, respectively; a total of 20,18 , and 15 minutes of data were obtained for the corresponding sleep states, respectively. From these segments, 20 randomly selected 20 -second samples were selected for analysis. Details regarding spectral and phase lag index (PLI) SEEG analyses and the corresponding statistics can be found in the Supplementary Material S1.

\section{Activation of VNS Decreases Global EEG Power}

During wakefulness, SC-VNS was associated with reduced overall power in all frequency bands except delta compared to the off setting, and RC-VNS was associated with reduced theta and alpha power (Fig. 2). During NREM sleep, SC-VNS was associated with reduced power in all frequency bands except high gamma, and RC-VNS was associated with reduced power in delta, theta, alpha, and low gamma. There appeared to be a trend toward further reduction in theta and alpha power in the awake state in the RC-VNS versus SC-VNS setting. Similar trends were also seen in the insulae but not in the cingula.

\section{Activation of VNS Increases EEG Alpha-Band Power During Sleep in the Anterior Cingulum}

VNS activation was associated with increased power in the alpha band during NREM sleep in the anterior cingulum, and the effects were more pronounced with SC-VNS than with RC-VNS. In contrast, during wakefulness, RCVNS was associated with increased low-gamma power.

\section{VNS Modulates Neural Synchrony}

Both SC- and RC-VNS were associated with decreased interhemispheric and hemispheric alpha band synchrony during wakefulness and sleep states and theta band synchrony during sleep states (Fig. 3). On the other hand, SCand RC-VNS were associated with increased interhemispheric and hemispheric delta and beta band synchrony during wakefulness. Compared to SC-VNS, RC-VNS was associated with significantly increased interhemispheric low- and high-gamma band synchrony. Furthermore, during wakefulness, RC-VNS was associated with increased low-gamma band synchrony in the frontal lobe and decreased theta band synchrony in the temporal lobe.

\section{VNS Decreases Hippocampal EEG Alpha-Band Power and Spike Counts}

In both awake and sleep states, RC- and SC-VNS were each associated with reduced power in the alpha frequency band in the bilateral hippocampi compared to the off setting (Fig. 2). Compared to SC-VNS, RC-VNS further reduced power in the alpha band in the bilateral hippocampi. In addition, RC-VNS decreased hippocampal spike counts compared to the off setting in the sleep state, and there was a similar trend in the awake state (Fig. 4).

\section{Discussion}

In this study, we assessed how two VNS duty cycles sequentially activated over 24-hour periods affected global and local neural dynamics. An increase in the duty cycle from $38 \%$ to $44 \%$ decreased the cumulative VNS on time from 8.56 hours/day (SC) to 6.72 hours/ day (RC). The acute responses following this increase in duty cycle were as follows: 1) selective decrease in EEG power in the alpha band in the epileptogenic hippocampi but not in the cingulum where there was an increase in alpha-band power-particularly by SC-VNS; 2) decreased interhemispheric and hemispheric alpha band synchrony that was independent of behavioral state (awake or sleep); and 3) increased interhemispheric and hemispheric gamma band synchrony during wakefulness. Activation of (RC) VNS decreased spike count within the epileptogenic hippocampus. Overall, our case demonstrates that titration of the VNS stimulation duration does not monotonically increase (or decrease) the 
Entry sites:
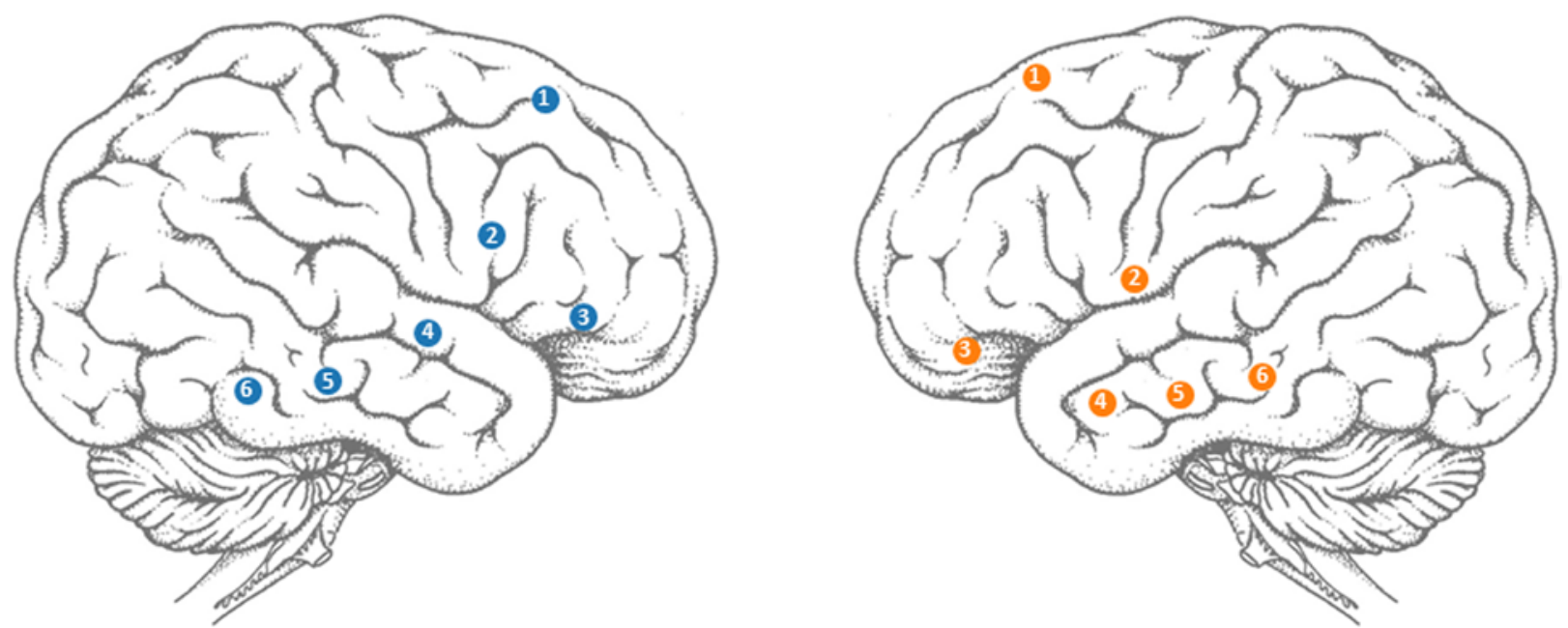

Targets:
(1) Anterior cingulate gyrus
(4) Amygdala
(2) 2 Posterior orbitofrontal gyrus
(3) Anterior orbitofrontal gyrus
(5) (5) Hippocampus (anterior)
6 6 Hippocampus (posterior)

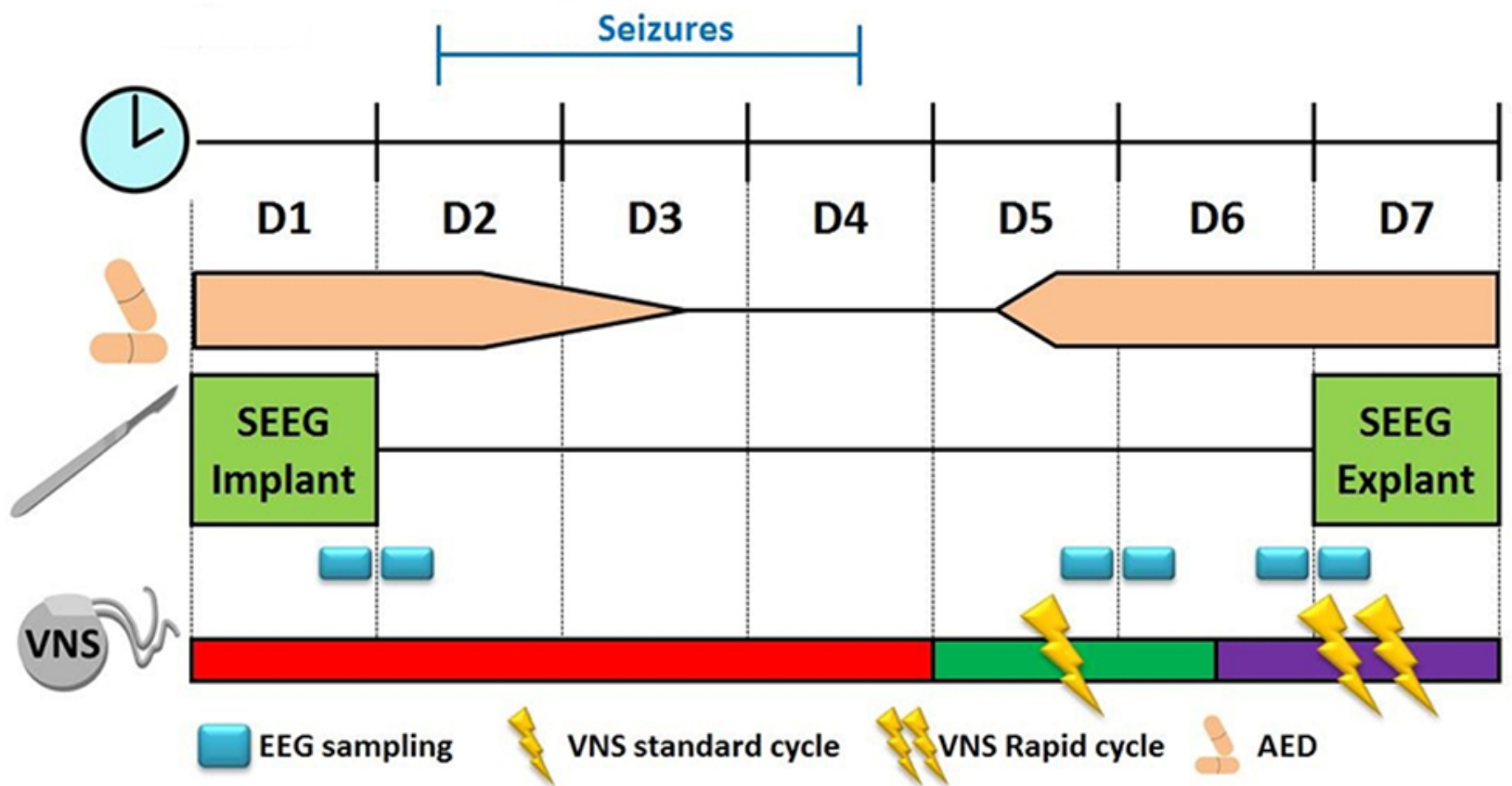

FIG. 1. Diagram (A) illustrating the entry sites and target locations of the 12 symmetrically implanted electrodes targeting the limbic structures bilaterally. Timeline of clinical course (B), highlighting the interval when seizures were captured and EEG activity was sampled relative to SEEG electrode implantation, anticonvulsant medication titration, SC-VNS, RC-VNS, and SEEG electrode explantation. $A E D=$ antiepileptic drug; $D 1, \ldots, D 7=$ day $1, \ldots$, day 7 . Figure is available in color online only. 

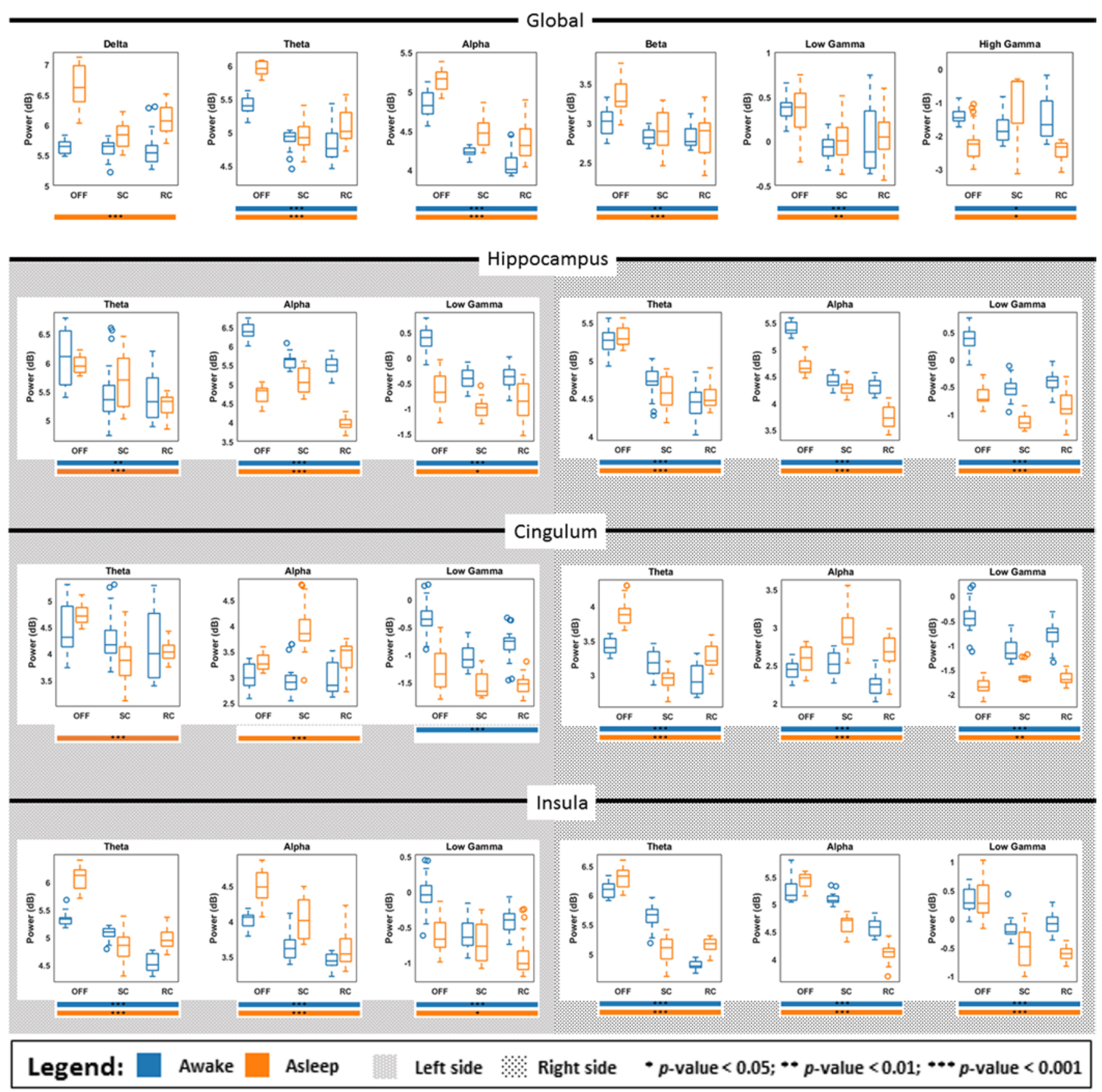

FIG. 2. Spectral analysis of the global, bilateral hippocampal, bilateral cingular, and bilateral insular electrodes in the awake and sleep states. Figure is available in color online only.

stimulation effects but rather modulates the regional and global dynamics differently.

The variable effects in global and regional cortical dynamics associated with different VNS stimulation parameters have been reported using functional imaging studies (e.g., functional MRI [fMRI]). ${ }^{13,18,20}$ For example, interleaved VNS-fMRI data demonstrated significant differences in activation patterns with stimulation at $20 \mathrm{~Hz}$ compared to stimulation at $5 \mathrm{~Hz}$ in patients with depression. ${ }^{18}$ However, cortical physiological studies evaluating
VNS dose-response effects are lacking. Prior studies have analyzed scalp and intracranial EEG characteristics to differentiate VNS responders from nonresponders and found that decreased global delta and alpha band synchrony was associated with an improved response to VNS therapy.,3

The reduction in hippocampal EEG power with SCand RC-VNS likely reflects a suppression of synaptic potentials and modulation of hippocampal excitability. The thalamus has been proposed to generate and modulate EEG alpha rhythm. ${ }^{25,26}$ Previous functional imaging 

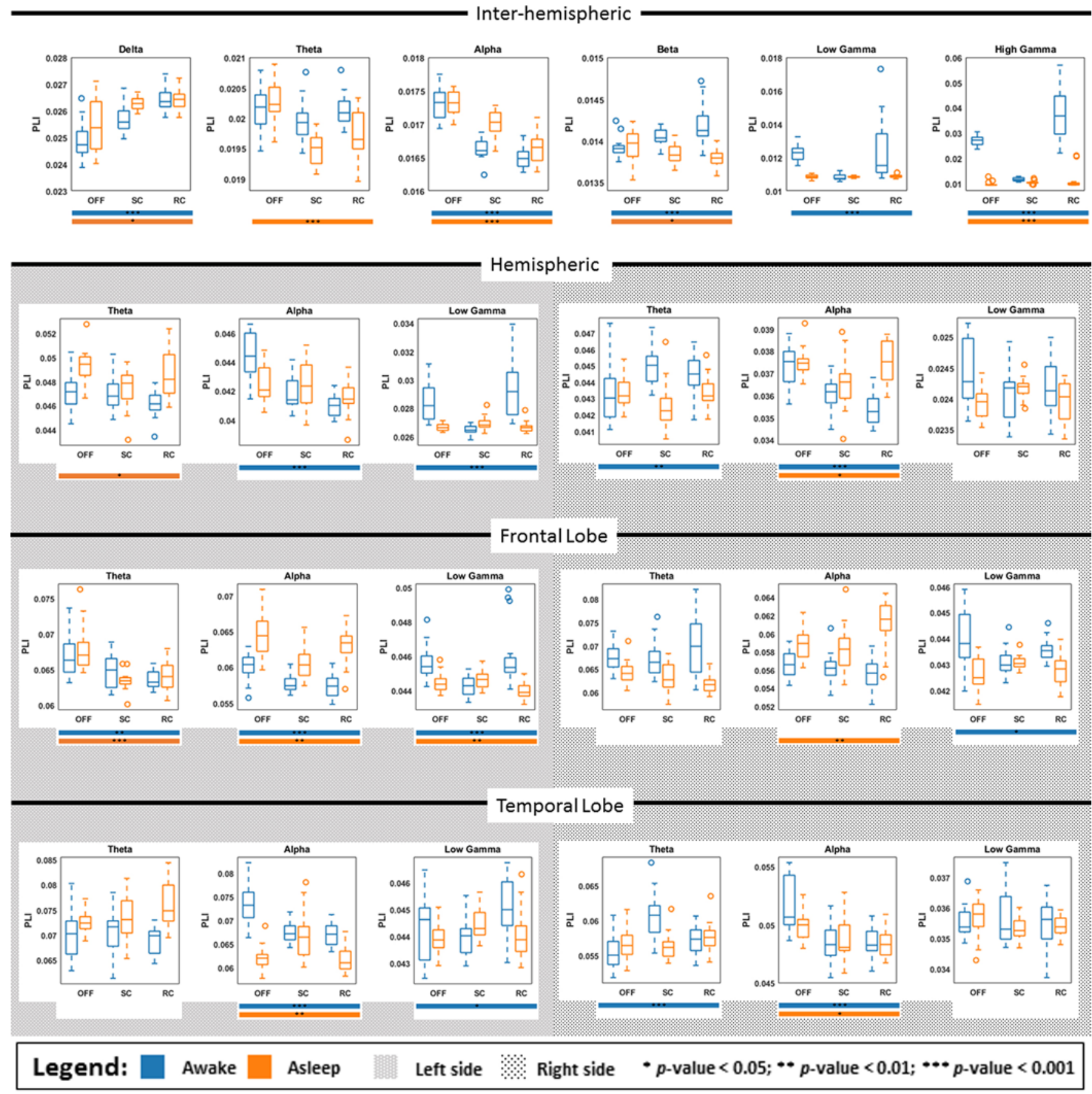

FIG. 3. Phase lag index analysis of the interhemispheric, bilateral hemispheric, bilateral frontal, and bilateral temporal electrodes in the awake and sleep states. Figure is available in color online only.

studies have demonstrated that VNS, in particular, RCVNS, decreases thalamic metabolic activity. ${ }^{24}$ Our finding of decreased hippocampal EEG power and decreased synchronization in the alpha band suggests that RC-VNS modulates thalamus and thalamocortical loops. ${ }^{24}$ Anecdotal reports suggest that daytime vigilance is improved following VNS therapy in patients with epilepsy. ${ }^{8}$ The increased synchrony in delta and gamma oscillations during wakefulness by RC-VNS suggests the potential of VNS in augmenting higher-order cognitive functions such as attention, motivation, and memory. ${ }^{12,14}$ Indeed, activation of VNS modulates the limbic network and has been demonstrated to improve performance of working memory by augmenting arousal and attention. ${ }^{4,23}$ Although the mechanistic underpinnings are not completely understood, stimulation of the vagus nerve has been shown to alter both the electrophysiological and metabolic profile of the forebrain, suggesting that the peripheral state of the body influences cognition. . $^{4,1521}$

Typically, phase synchronization is measured on short- 

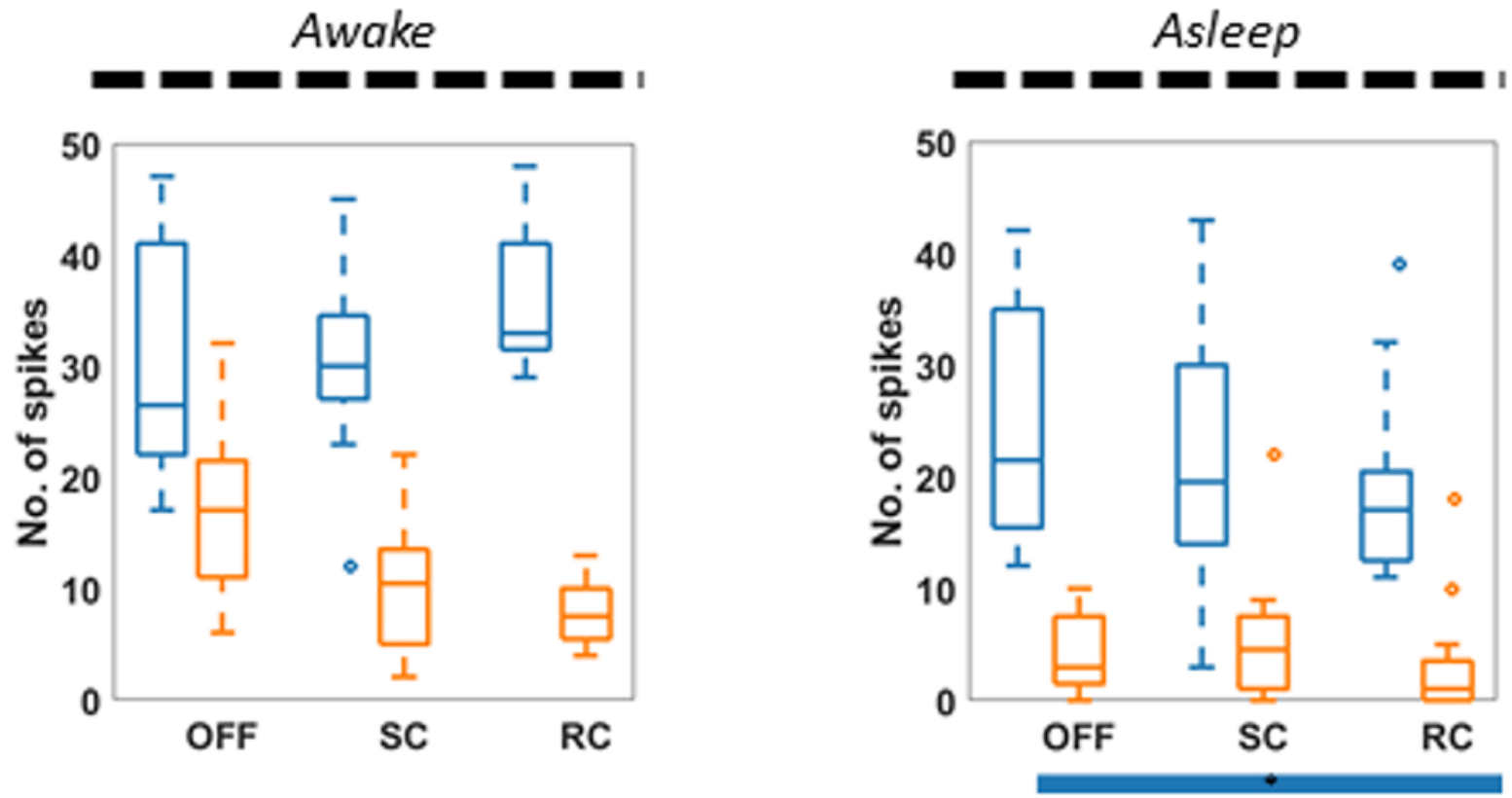

\section{Legend: $\square$ Right hippocampus}

\section{Left hippocampus}

\section{$\bullet p$-value $<0.05 ; \cdots p$-value $<0.01 ; \cdots p$-value $<0.001$}

FIG. 4. Hippocampal spike count analysis in the awake and sleep states. Figure is available in color online only.

er intervals than the 20-second interval used in our analysis. A longer time interval was chosen because the present case was focused on the analysis of effects that persist beyond the duration of stimulation (i.e., tonic effects). On the other hand, phasic effects would occur on the order of milliseconds-certainly within the subsecond time frame. The use of very long time intervals (e.g., 60 seconds) can result in unreliable results. ${ }^{11}$ Therefore, selection of the temporal window duration is a tradeoff between reliable output and the duration of the event of interest.

\section{Limitations}

These results, having been derived from a single patient, may not generalize to all VNS implant patients. In addition, the neuromodulatory effects of SC- and RC-VNS were assessed in the acute inpatient setting; however, longterm follow-up is needed to determine the anticonvulsive effects of RC-VNS in this patient. The limited availability of EEG data presents the potential for sampling bias despite adherence to strict behavioral and EEG criteria for selection of awake and sleep states. Unfortunately, longer durations of sampling were unobtainable as a consequence of these criteria. Nonetheless, the samples obtained for analysis were noncontiguous, which decreases the risk of sampling bias. Furthermore, whereas the typical longrange conduction delay is on the order of tens of milliseconds, our use of longer segments for analysis is likely to detect sustained synchronization, which is of interest in this study as the SC- and RC-VNS are each a continuously cycling stimulus. ${ }^{6}$ We performed a focused spectral analysis of the hippocampi, cingula, and insulae as the patient's seizures originated from the bilateral hippocampi and VNS has been shown to modulate the limbic network. In addition, the hippocampus plays a crucial role in temporal lobe epilepsy; therefore, we investigated the dose-response effects of VNS on the hippocampus. ${ }^{1}$

Spikes may result in spurious oscillations, thereby leading to significant change in synchrony measures. However, spikes last less than a second $(<70 \mathrm{msec})$. To reduce the confounding effects of spikes, we selected EEG segments that lack any periodic runs of spikes. In addition, we visually screened the epileptogenic zones to select spike-free epochs for analysis. Despite our efforts, a few spikes may have been present in some of the epochs, but these are unlikely to have a confounding effect given that spikes are transient and we selected multiple noncontiguous 20 -second epochs.

It is important to consider that our findings may reflect the cumulative effects of VNS rather than the effects of the change in VNS parameters from SC to RC. In other words, we assessed the combined dose-response and temporal effects of VNS on neural modulation. It is interesting to note that not all effects were monotonic. Thus, if the observed changes were secondary to cumulative stimulation effects alone, then it would imply that VNS reverses both global and regional power and synchrony in certain frequency bands in a time-dependent manner. Unfortunately, our trial was not designed to specifically test this hypothesis, which would be better tested with a crossover design. 


\section{Conclusions}

VNS may induce a complex modulation of the corticosubcortical network with titration of duty cycles. RC-VNS can induce robust suppression of EEG power in epileptogenic hippocampi and insulae and global and hemispheric alpha band desynchronization. Further studies are needed to investigate the mechanism behind VNS neural modulation.

\section{References}

1. Avoli M: The epileptic hippocampus revisited: back to the future. Epilepsy Curr 7:116-118, 2007

2. Bartolomei F, Bonini F, Vidal E, Trébuchon A, Lagarde S, Lambert I, et al: How does vagal nerve stimulation (VNS) change EEG brain functional connectivity? Epilepsy Res 126:141-146, 2016

3. Bodin C, Aubert S, Daquin G, Carron R, Scavarda D, McGonigal A, et al: Responders to vagus nerve stimulation (VNS) in refractory epilepsy have reduced interictal cortical synchronicity on scalp EEG. Epilepsy Res 113:98-103, 2015

4. Clark KB, Naritoku DK, Smith DC, Browning RA, Jensen RA: Enhanced recognition memory following vagus nerve stimulation in human subjects. Nat Neurosci 2:94-98, 1999

5. Connor DE Jr, Nixon M, Nanda A, Guthikonda B: Vagal nerve stimulation for the treatment of medically refractory epilepsy: a review of the current literature. Neurosurg Focus 32(3):E12, 2012

6. David O, Bastin J, Chabardès S, Minotti L, Kahane P: Studying network mechanisms using intracranial stimulation in epileptic patients. Front Syst Neurosci 4:148, 2010

7. DeGiorgio CM, Thompson J, Lewis P, Arrambide S, Naritoku D, Handforth A, et al: Vagus nerve stimulation: analysis of device parameters in 154 patients during the long-term XE5 study. Epilepsia 42:1017-1020, 2001

8. Galli R, Bonanni E, Pizzanelli C, Maestri M, Lutzemberger L, Giorgi FS, et al: Daytime vigilance and quality of life in epileptic patients treated with vagus nerve stimulation. Epilepsy Behav 4:185-191, 2003

9. George MS, Nahas Z, Li X, Kozel FA, Anderson B, Yamanaka K, et al: Novel treatments of mood disorders based on brain circuitry (ECT, MST, TMS, VNS, DBS). Semin Clin Neuropsychiatry 7:293-304, 2002

10. Gschliesser V, Högl B, Frauscher B, Brandauer E, Poewe W, Luef G: Mode of vagus nerve stimulation differentially affects sleep related breathing in patients with epilepsy. Seizure 18:339-342, 2009

11. Hardmeier M, Hatz F, Bousleiman H, Schindler C, Stam C, Fuhr P: P30: Test-retest reliability and inter-subject variability of the Phase Lag Index (PLI), a measure of functional connectivity in EEG analysis. Clin Neurophysiol 125:S56-S57, 2014

12. Hays SA, Rennaker RL, Kilgard MP: Targeting plasticity with vagus nerve stimulation to treat neurological disease. Prog Brain Res 207:275-299, 2013

13. Henry TR, Bakay RAE, Pennell PB, Epstein CM, Votaw JR: Brain blood-flow alterations induced by therapeutic vagus nerve stimulation in partial epilepsy: II. Prolonged effects at high and low levels of stimulation. Epilepsia 45:1064-1070, 2004

14. Kilgard MP: Harnessing plasticity to understand learning and treat disease. Trends Neurosci 35:715-722, 2012

15. Ko D, Heck C, Grafton S, Apuzzo ML, Couldwell WT, Chen $\mathrm{T}$, et al: Vagus nerve stimulation activates central nervous system structures in epileptic patients during PET $\mathrm{H}_{2}{ }^{15} \mathrm{O}$ blood flow imaging. Neurosurgery 39:426-431, 1996
16. Labar D: Vagus nerve stimulation for 1 year in 269 patients on unchanged antiepileptic drugs. Seizure 13:392-398, 2004

17. Larsen LE, Wadman WJ, Marinazzo D, van Mierlo P, Delbeke J, Daelemans S, et al: Vagus nerve stimulation applied with a rapid cycle has more profound influence on hippocampal electrophysiology than a standard cycle. Neurotherapeutics 13:592-602, 2016

18. Lomarev M, Denslow S, Nahas Z, Chae JH, George MS, Bohning DE: Vagus nerve stimulation (VNS) synchronized BOLD fMRI suggests that VNS in depressed adults has frequency/dose dependent effects. J Psychiatr Res 36:219-227, 2002

19. Marangell LB, Rush AJ, George MS, Sackeim HA, Johnson CR, Husain MM, et al: Vagus nerve stimulation (VNS) for major depressive episodes: one year outcomes. Biol Psychiatry 51:280-287, 2002

20. Mu Q, Bohning DE, Nahas Z, Walker J, Anderson B, Johnson KA, et al: Acute vagus nerve stimulation using different pulse widths produces varying brain effects. Biol Psychiatry 55:816-825, 2004

21. O'Brien JH, Pimpaneau A, Albe-Fessard D: Evoked cortical responses to vagal, laryngeal and facial afferents in monkeys under chloralose anaesthesia. Electroencephalogr Clin Neurophysiol 31:7-20, 1971

22. Reed CM, Birch KG, Kamiński J, Sullivan S, Chung JM, Mamelak AN, et al: Automatic detection of periods of slow wave sleep based on intracranial depth electrode recordings. J Neurosci Methods 282:1-8, 2017

23. Sun L, Peräkylä J, Holm K, Haapasalo J, Lehtimäki K, Ogawa KH, et al: Vagus nerve stimulation improves working memory performance. J Clin Exp Neuropsychol 39:954964, 2017

24. Van Laere K, Vonck K, Boon P, Brans B, Vandekerckhove T, Dierckx R: Vagus nerve stimulation in refractory epilepsy: SPECT activation study. J Nucl Med 41:1145-1154, 2000

25. Vonck K, Boon P, Van Roost D: Anatomical and physiological basis and mechanism of action of neurostimulation for epilepsy. Acta Neurochir Suppl 97:321-328, 2007

26. Vonck K, Van Laere K, Dedeurwaerdere S, Caemaert J, De Reuck J, Boon P: The mechanism of action of vagus nerve stimulation for refractory epilepsy: the current status. J Clin Neurophysiol 18:394-401, 2001

\section{Disclosures}

Dr. Pati has been a consultant for NeuroPace.

\section{Author Contributions}

Conception and design: Ilyas, Toth, Pati. Acquisition of data: Ilyas, Toth, Pati. Analysis and interpretation of data: Ilyas, Toth. Drafting the article: Ilyas. Critically revising the article: all authors. Reviewed submitted version of manuscript: all authors. Approved the final version of the manuscript on behalf of all authors: Ilyas. Statistical analysis: Ilyas. Administrative/technical/ material support: Riley, Pati. Study supervision: Riley, Pati.

\section{Supplemental Information \\ Online-Only Content}

Supplemental material is available with the online version of the article.

Supplementary Material S1. https://thejns.org/doi/suppl/10. 3171/2018.6.JNS18735.

\section{Correspondence}

Adeel Ilyas: University of Alabama at Birmingham, AL. adeelilyas@uabmc.edu. 